\title{
Simultaneous Multiple Channel All-Optical NRZ to CSRZ and RZ to CSRZ Format Conversion Using an SOA-NOLM
}

\author{
M. H. A. Wahid ${ }^{1}$ \\ ${ }^{1}$ Semiconductor Photonics and Integrated Lightwave Systems(SPILS), \\ School of Microelectronic Engineering, Universiti Malaysia Perlis, \\ Pauh Putra Campus, 02600 Arau, Perlis. \\ M. M. Nahas ${ }^{2}$, R. A. Ibbotson ${ }^{2}$ and K. J. Blow ${ }^{2}$ \\ ${ }^{2}$ Photonics Research Group, School of Engineering and Applied Science, \\ Aston University, Aston Triangle, Birmingham B4 7ET, United Kingdom.
}

\begin{abstract}
The optical conversion bandwidth for an all-optical modulation format converter, based on a semiconductor laser amplifier in a nonlinear optical loop mirror (SOA-NOLM), is investigated. $4 \times 10 \mathrm{Gbit} / \mathrm{s}$ channels are all-optically converted between both non-return-to-zero (NRZ) and return-to-zero (RZ) format to carrier-suppressed return-to-zero (CSRZ). WDM transmission of the converted signals over a $194 \mathrm{~km}$ fibre span is then demonstrated. The receiver sensitivity for the converted four wavelengths is measured and compared after transmission.

(C) 2014 Elsevier B.V. All rights reserved.

Keywords: SOA-NOLM; CSRZ format conversion; WDM transmission
\end{abstract}




\section{Introduction}

The carrier-suppressed return-to-zero (CSRZ) modulation format has aroused interest due to its superior tolerance to nonlinear and dispersion impairments than that of the more conventional non-return-to-zero (NRZ) and return-to-zero (RZ) formats [1]. CSRZ reduces the main carrier component, which carries the majority of the signal power for the RZ format, and narrows the optical spectrum yielding a format that has a higher spectral efficiency and is more tolerant to chromatic dispersion. For WDM applications, CSRZ has been shown to be less sensitive to four-wave-mixing (FWM) between the WDM channels [2], where there are no carrier components in the transmitted signals. However, CSRZ signal generation is more complex than the conventional NRZ and RZ since at least one additional modulator is required [3]. This modulator can be a $\mathrm{LiNbO}_{3}$ Mach-Zehnder modulator, which is an electro-optic device. Recently, all-optical devices have been considered to be of key importance in future all-optical networks where the signal remains in the optical domain all over the network without being converted to electronics [4]. This indeed can increase the cost effectiveness of the WDM network. Therefore, all-optical signal processing including switching, demultiplexing, signal regeneration and format conversion have recently received much interest. Here we investigate an alloptical format conversion scheme [5] capable of converting both NRZ and RZ to CSRZ. In particular we focus on the performance of an optical loop mirror based format converter that would be suitable for use within a WDM network, as opposed to conventional CSRZ transmitters. Cascaded sum and difference frequency generation in periodically poled lithium niobate waveguide has been 
demonstrated that offers CSRZ but with very complicated setup and low conversion efficiency [6]. Optical sampling on NRZ signals based on cross gain modulation has also reported to produce CSRZ [7]. However, it requires a mode locked laser source at full bit rate which is more complex than the half rate sinewave source.

Utilising a semiconductor optical amplifier (SOA) based fibre loop mirror for NRZ and RZ to CSRZ conversion was first shown in [5] for a single channel, no transmission was demonstrated. Since it is important to establish the suitability of converted optical signals for onward transmission, we also include some optical transmission in our investigation. The conversion is implemented by injecting a control beam to modulate the saturation, and hence the refractive index, of the SOA. The control beam is sinusoidal in optical power at half the data channel clock frequency. The mean optical control power ideally biases the loop to a differential phase shift of $2 \pi$ and the sinusoidal amplitude should also give a phase swing of $2 \pi$. At the output of the loop mirror, when the clockwise and the counter clockwise signals are recombined the transmission is $100 \%$ for all the data pulses but their phase is alternately zero and $\pi$. Therefore the input NRZ or $R Z$ data signal is converted into a CSRZ signal. If the control pulse is absent, the clockwise and counter clockwise signals will destructively interfere at the output port. If the bias is adjusted from $2 \pi$ to $\pi$, the interference will be constructive; hence the input NRZ or RZ signal is switched out of the SOA-NOLM without changing its modulation format [8].

In this letter, we investigate experimentally the feasibility of a fibre loop mirror based CSRZ format converter for $4 \times 10$ Gbit/s WDM conversion and 
transmission. We explore the available optical conversion bandwidth for this device and its limitations. The BER performance for the converted WDM channels against their original NRZ and RZ counterparts after transmission over a SSMF, DCF fibre span is also given.

\section{Device Modelling}

Fig.1 depicts the SOA-NOLM converter with the transmission system used in the study. The CSRZ converter was simulated for a single channel and a WDM system with four input channels. It was modelled using OptSim whereby all components were the same as given in Fig.1. The calculated eye diagrams for the single channel in Fig.2 show the converted signal when the input has an RZ modulation format, Fig.2a, and an NRZ modulation format, Fig.2b. We see that the CSRZ pulses possess the required RZ signal shape in each case. Although both derived signals are CSRZ they are clearly not identical to each other. This was also observed in the experiments which were shown in the respective inset. The two converted CSRZ data streams have different pulse widths and phase profiles reflecting the properties of the original source they are derived from. The signal derived from the NRZ source has a pulse shape determined by the

temporal modulation of the converter whereas the signal derived from the RZ source has a pulse shape determined by the product of this temporal modulation and the RZ pulse shape. In the limit that the RZ pulse width is short compared to the modulation window then the output pulse would be the same width as the input $R Z$ pulse. The full width at half-maximum (FWHM) for the $R Z$ to $C S R Z$ case is about 40 ps while for the NRZ to CSRZ it is 60 ps. The BER calculations show an error rate of $10^{-10}$ can be achieved both before and after the format 
conversion. Fig.3a and Fig.3b show WDM system with spectral spacing of 0.8 $\mathrm{nm}$ by simulation and experiment respectively. The same observations were recorded for $1.8 \mathrm{~nm}$ and $3.2 \mathrm{~nm}$ spectral spacing. Thus, the conversion will take place as long as the signals fall within SOA's bandwidth irrespective of number of wavelengths. The reflected signal is always the same format as the input signal in all the simulations and experiments.

\section{Experimental setup}

The CSRZ conversion and transmission was set up according to Fig.1. Four CW laser sources, starting at $1554.7 \mathrm{~nm}$ with an even separation of $100 \mathrm{GHz}$, were modulated with a $2^{31}-1$ pseudo random bit sequence (PRBS) via one $\mathrm{LiNbO}_{3}$ modulator for the NRZ format, and two $\mathrm{LiNbO}_{3}$ modulators for the $\mathrm{RZ}$ format. The pulse pattern generator generating the PRBS sequence was driven by an external clock reference of $10.708 \mathrm{GHz}$, suitable for the application of forward error correction to a $10 \mathrm{Gbit} / \mathrm{s}$ stream although none was applied. The resultant data stream was amplified and launched into the converter. The SOANOLM comprised of a 50/50 coupler with polarisation controllers (PCs) on each arm, a 50/50 coupler on the clockwise path and an SOA offset from the loop centre by 140ps by a variable optical delay line on the clockwise arm. The SOA provided a peak fibre-to-fibre gain of $20 \mathrm{~dB}$, and a saturation output power of $3.85 \mathrm{dBm}$ when biased at $200 \mathrm{~mA}$. A CW DFB laser with a wavelength of 1542.3 $\mathrm{nm}$ was modulated at $5.354 \mathrm{GHz}$, delayed and coupled, via the $50 / 50$ intra-loop coupler, into the SOA-NOLM at a power of $3.2 \mathrm{dBm}$. A variable optical delay line, to adjust the control pulse arrival time relative to the data, controlled the phase seen by the clockwise and counter clockwise data at the SOA. On the output of 
the SOA-NOLM a WDM coupler was used to extract the $1542.3 \mathrm{~nm}$ control wavelength whilst allowing the data to propagate along the fibre span. The spectral resolution of the spectrum analyser which was $2 \mathrm{~nm}$ limited the observation of sharp peaks as depicted in Fig.3a. For the SOA-NOLM bandwidth measurements, the WDM coupler was replaced by a fibre Bragg grating (FBG) with a $3 \mathrm{~dB}$ bandwidth of $1.1 \mathrm{~nm}$ and isolation of $19.8 \mathrm{~dB}$ to reflect the control wavelength, which was prevented from re-entering the SOA-NOLM by a circulator.

After conversion, the data propagated along a $194 \mathrm{~km}$ fibre span, consisting of two dispersion managed sections of approximately $40 \mathrm{~km}$ of SSMF, $16 \mathrm{~km}$ of DCF and $40 \mathrm{~km}$ SSMF. The SSMFs have an attenuation coefficient of $0.2 \mathrm{~dB} / \mathrm{km}$ and dispersion of $17 \mathrm{ps} / \mathrm{nm} / \mathrm{km}$ at $1550 \mathrm{~nm}$, while the DCF units each had a negative dispersion of $1385 \mathrm{ps} / \mathrm{nm}$ and an insertion loss of $7.9 \mathrm{~dB}$. The losses were compensated using two C-band Erbium-doped fibre amplifier (EDFA) repeaters within each section. The EDFAs denoted 1, in Fig. 1, have a $30 \mathrm{~dB}$ maximum small signal gain, while the EDFAs denoted 2 have a $40 \mathrm{~dB}$ maximum small signal gain and both types of EDFA have a $5 \mathrm{~dB}$ noise figure. An ASE filter with an insertion loss of $3 \mathrm{~dB}$ was used before the pre-receiver amplifier to remove the ASE gain peak at $1530 \mathrm{~nm}$. A tunable grating filter, with a $3 \mathrm{~dB}$ bandwidth of $0.24 \mathrm{~nm}$ and a $7 \mathrm{~dB}$ insertion loss, was used prior to the bit error rate (BER) analyser and the clock recovery unit in order to isolate an individual channel for error detection.

\section{Results and discussion}

Fig. 4 depicts the spectral outputs taken before the transmission span for 
four arbitrary wavelengths spanning the available conversion bandwidth of the converter for RZ to CSRZ conversion. The lowest and the highest wavelengths at which the signal is fully converted to CSRZ with BER $\leq 10^{-9}$ are $1531.4 \mathrm{~nm}$ and $1562.2 \mathrm{~nm}$, respectively. This bandwidth is limited by the active region of the SOA as well as the wavelength of the control pulse used to saturate the SOA in the NOLM. Even with the additional $19.8 \mathrm{~dB}$ isolation provided by the fibre Bragg grating, a peak $2 \mathrm{~dB}$ above the noise floor at $1542.3 \mathrm{~nm}$ can be clearly seen. Such partial suppression of the switching signal reduces the conversion region by approximately $1 \mathrm{~nm}$ either side of the control signal resulting in a total available bandwidth of $28.8 \mathrm{~nm}$ (depicted as the shaded regions in Fig. 4). Pulse width of the converted signals was observed to be 40 ps for RZ to CSRZ and 50 ps for NRZ to CSRZ. Higher control pulse is necessary if more wavelengths to be added in this conversion. However, it depends on the input power of each wavelength. This converter could work at higher speed for instance $40 \mathrm{Gbs}^{-1}$ as long as the recovery rate for the SOA allows suitable amount of phase shift in the system.

We will now show that the converted pulses do not suffer from excess chirping or pulse distortion and are therefore suitable for onward transmission in the network. In the transmission experiments, a WDM coupler was used in place of the fibre Bragg grating, as mentioned above. This increased the suppression of the control pulse by an additional $17.2 \mathrm{~dB}$ albeit at the expense of available bandwidth, as the $3 \mathrm{~dB}$ cut-off for the transmission port on the WDM coupler was at $1551 \mathrm{~nm}$. Ideally a device with the suppression performance of the WDM coupler but with the bandwidth of the fibre Bragg grating should be used. Optical 
signal to noise ratio, OSNR can be increased by filtering more amount of the control pulse power at the converter which acts as the transmitter. Further improve in the signal quality or OSNR might also be expected by using suitable ASE filter in the transmission channel. However, the system used sufficiently high power ( 4.6 dBm per channel) in all formats to ensure nonlinear effects during transmission. This will be useful in comparing the tolerance of the converted and unconverted signals towards nonlinear effects. For consistency, the comparisons between the converted and unconverted signal propagation were all performed with the signals propagating through the SOA-NOLM before being transmitted over the fibre span. This means that all signals had the same environmental condition where any impairment introduced by the loop mirror was common to all measurements. In the case of the unconverted NRZ/RZ signal, the SOA-NOLM was biased at a differential phase shift of $\pi$ with no control pulses applied. The SOA-NOLM thus switched the input NRZ or RZ signal out without changing its modulation format as mentioned above.

Fig. 5 shows the BER results after transmission over the $194 \mathrm{~km}$ span for the four WDM channels with different formats, where Fig. 5(a) is the NRZ signal and its converted CSRZ version, and Fig. $5(\mathrm{~b})$ is the RZ signal and its converted CSRZ version. A received power of $-17.9 \mathrm{dBm}$ per channel was required to achieve a BER of $10^{-9}$ for the NRZ format, whereas the converted format required a lower power of $-18.8 \mathrm{dBm}$ to attain the same error rate. For the $\mathrm{RZ}$ case, the received power required for $10^{-9} \mathrm{BER}$ was $-22 \mathrm{dBm}$, while for the converted format it was $-22.5 \mathrm{dBm}$. The slope of the BER-power curve for both converted carrier-suppressed formats decays slower than that of their original counterparts. 
This might be due to the response of the photo detector at the receiver. At a BER of $10^{-5}$, the improvement in required received power has increased from $0.9 \mathrm{~dB}$ to $1.2 \mathrm{~dB}$ for $\mathrm{NRZ}$ and from $0.5 \mathrm{~dB}$ to $0.9 \mathrm{~dB}$ for RZ. This would imply that the receiver sensitivity improvement at $10^{-9} \mathrm{BER}$ could be further increased if forward error correction (FEC) is used to correct errors at the level of a $10^{-5}$ error rate. In addition, the receiver sensitivities at the output of the SOA-NOLM were measured without transmission for all formats using a single-channel at 1555.2 $\mathrm{nm}$. It was found that in the case of NRZ to CSRZ, the received power required for the converted CSRZ for $10^{-9} \mathrm{BER}$ was less than that for the unconverted NRZ by $0.5 \mathrm{~dB}$. In the case of RZ to CSRZ, the converted and unconverted formats had almost the same required power for $10^{-9} \mathrm{BER}$. Therefore we conclude the results obtained are attributed to the format conversion. This is most likely due to the change in the initial pulse format i.e. the CSRZ we derive from RZ is different to the CSRZ we derive from NRZ as explained in section 2. This implies that the transmission improved the sensitivity of the CSRZ format by $0.4 \mathrm{~dB}$ in the case of $\mathrm{NRZ}$, and $0.5 \mathrm{~dB}$ in the case of RZ format. However, as this system employed $194 \mathrm{~km}$ dispersion-managed fibre, using the SSMF-DCF configuration, and 100 $\mathrm{GHz}$ channel spacing; it should not show large transmission penalties for the formats studied if those converted signals were close to ideal. Since small transmission penalties were indeed found, we conclude that the converted pulses do not suffer from unwanted phase modulation or pulse distortion. More considerable transmission penalties would be expected if the transmission configuration is modified to demonstrate significant four-wave mixing (FWM) by using lower local dispersion, e.g. DSF, or higher inter-channel interaction by 
using a lower channel spacing.

\section{Conclusion}

All-optical format conversion between NRZ to CSRZ and conversion between RZ to CSRZ was experimentally demonstrated, using an SOA-NOLM. The bandwidth of the converter was shown to be $28.8 \mathrm{~nm}$ and was also shown to be capable of simultaneous conversion of 4 channels. $4 \times 10 \mathrm{Gbit} / \mathrm{s}$ WDM transmission over a $194 \mathrm{~km}$ fibre span after format conversion was also demonstrated thus showing the converted signals were of good quality. The comparisons were all performed with the loop mirror in place so that any signal or noise degradation introduced by the SOA-NOLM was common to all the measurements. The receiver sensitivity for the converted four wavelengths was compared with the sensitivity for the original NRZ and RZ wavelengths.

\section{Acknowledgements:}

The authors would like to thank Aston University for supporting the experimental works and Universiti Malaysia Perlis in the simulation results. 


\section{References:}

[1] Y. Miyamoto, A. Hirano, K. Yonenaga, A. Sano, H. Toba, K. Murata, O. Mitomi, Electron. Lett. 35 (1999) 2041.

[2] T. Tokle, C. Peucheret, P. Jeppesen, Opt. Commun. 225 (2003) 79.

[3] J. Yan , M. Chen, S. Xie, and B. Zhou, Opt. Commun. 231 (2004) 175.

[4] B. Mukherjee, Optical Communication Networks, McGraw-Hill, New York, 1997.

[5] W. Li, M. Chen, Y. Dong, S. Xie, IEEE Photon. Technol. Lett.16 (2004) 203.

[6] J. Wang and Q. Sun, Electronics Letters, 45 (2009) 420.

[7] T. Silveira, A. Ferreira, A. Teixeira, and P. Monteiro, IEEE Photon. Technol. Lett. 20 (2008) 1597.

[8] R. J. Manning, A. D. Ellis, A. J. Poustie, K. J. Blow, J. Opt. Soc. Am. B 14 (1997) 3204. 


\section{Figure captions:}

Fig. 1. Experimental configuration of the CSRZ-converter and $194 \mathrm{~km}$ fibre span.

Fig. 2. The simulated eye diagrams for (a) RZ to CSRZ and (b) NRZ to CSRZ.

Fig. 3. The CSRZ spectra in WDM system for (a) simulation and (b) experiment.

Fig. 4. The spectral outputs taken before the transmission span for four arbitrary wavelengths within the $B E R<10^{-9}$ conversion bandwidth of the RZ to CSRZ converter. The full conversion bandwidth is depicted by the two shaded regions.

Fig. 5. The BER versus received power after $194 \mathrm{~km}$ transmission for different converted signal formats: (a) NRZ (solid) and its converted CSRZ (dashed) and (b) RZ (solid) and its converted CSRZ (dashed).

$$
\begin{array}{ll}
\square & \lambda=1554.7 \mathrm{~nm} \\
\diamond & \lambda=1555.5 \mathrm{~nm} \\
\Delta & \lambda=1556.3 \mathrm{~nm} \\
\times & \lambda=1557.1 \mathrm{~nm}
\end{array}
$$


Figure 1

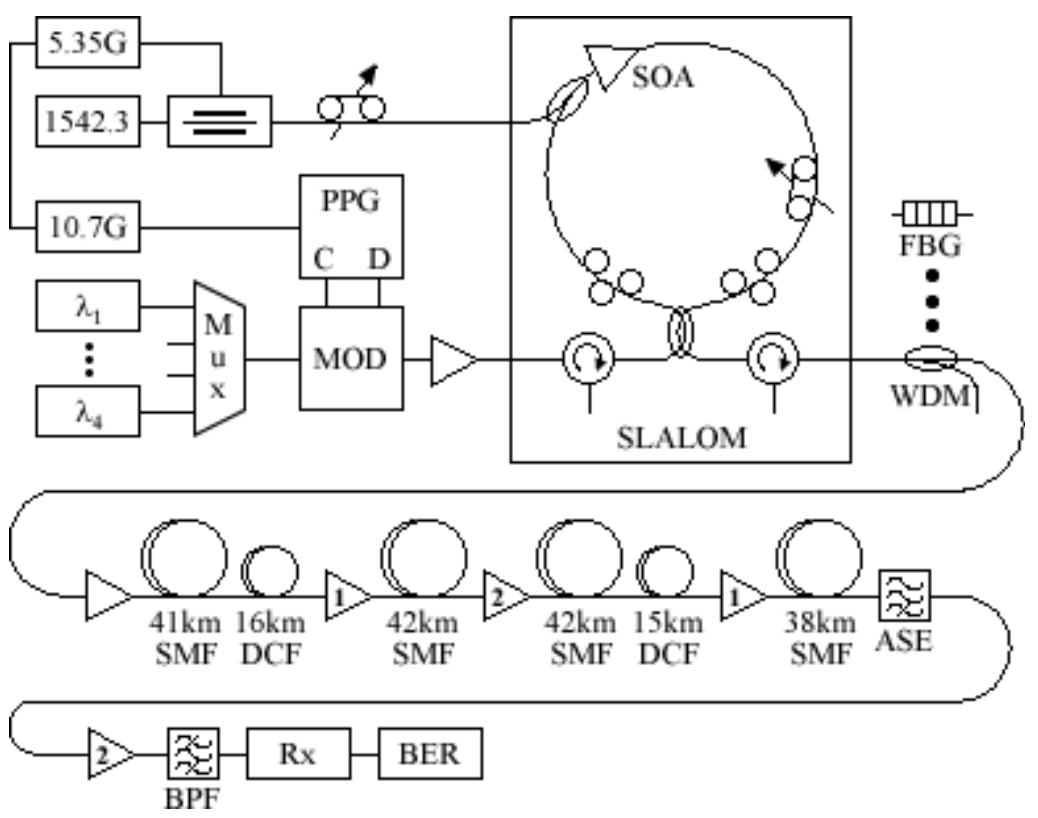


Figure 2

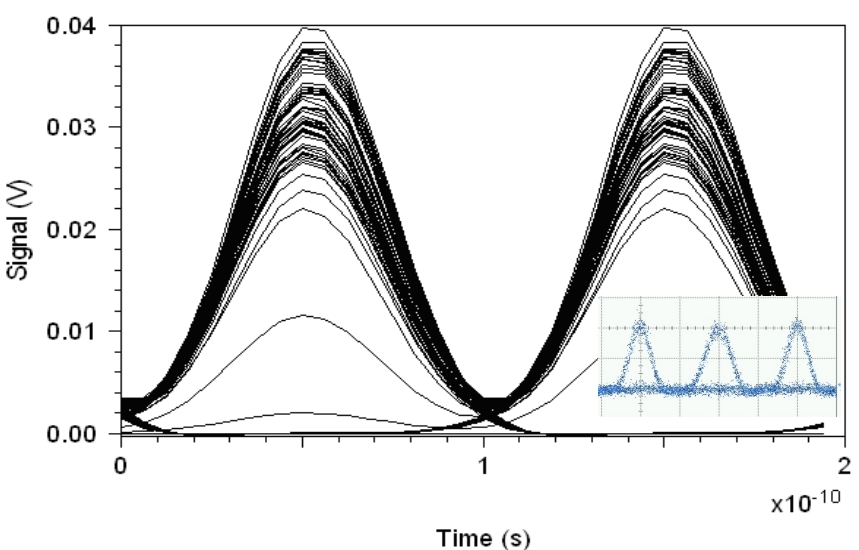

a

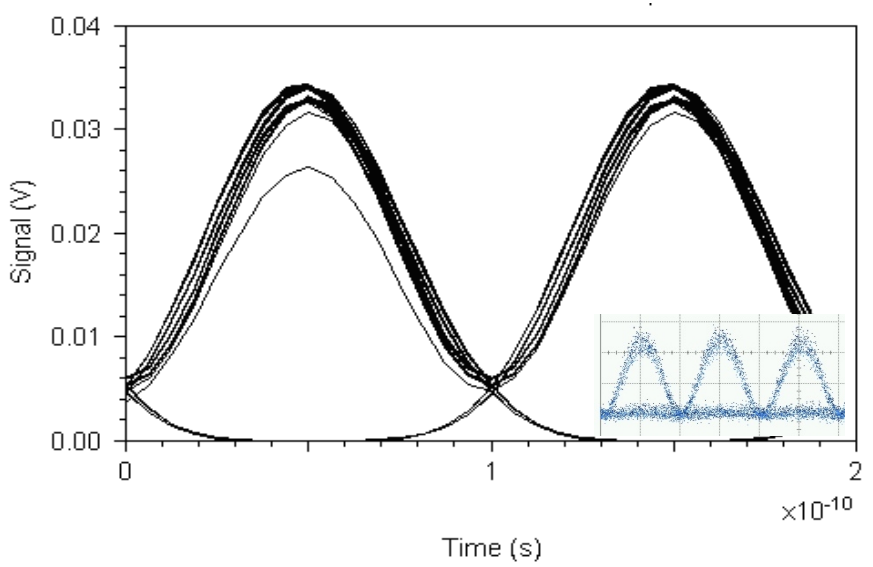

b 
Figure 3

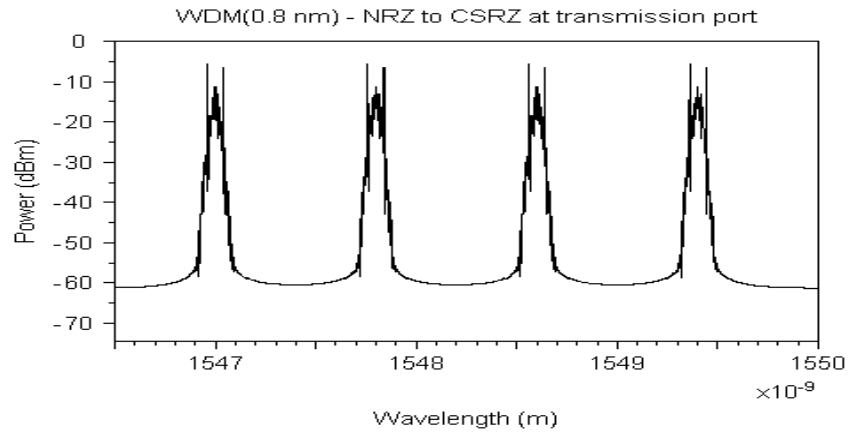

a

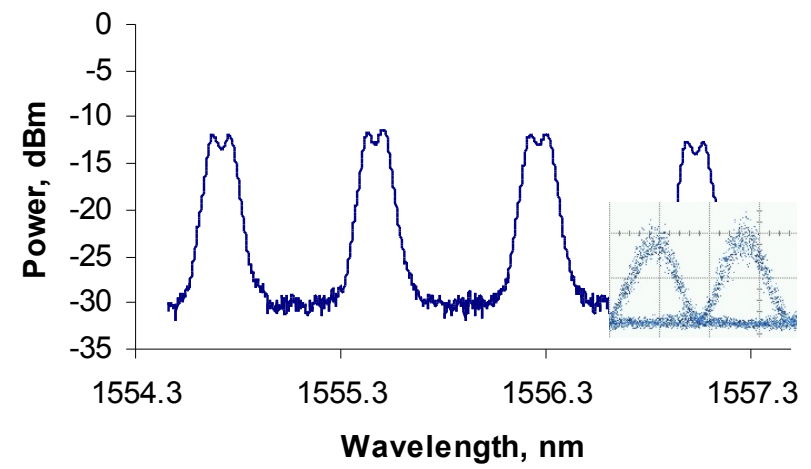

b 
Figure 4

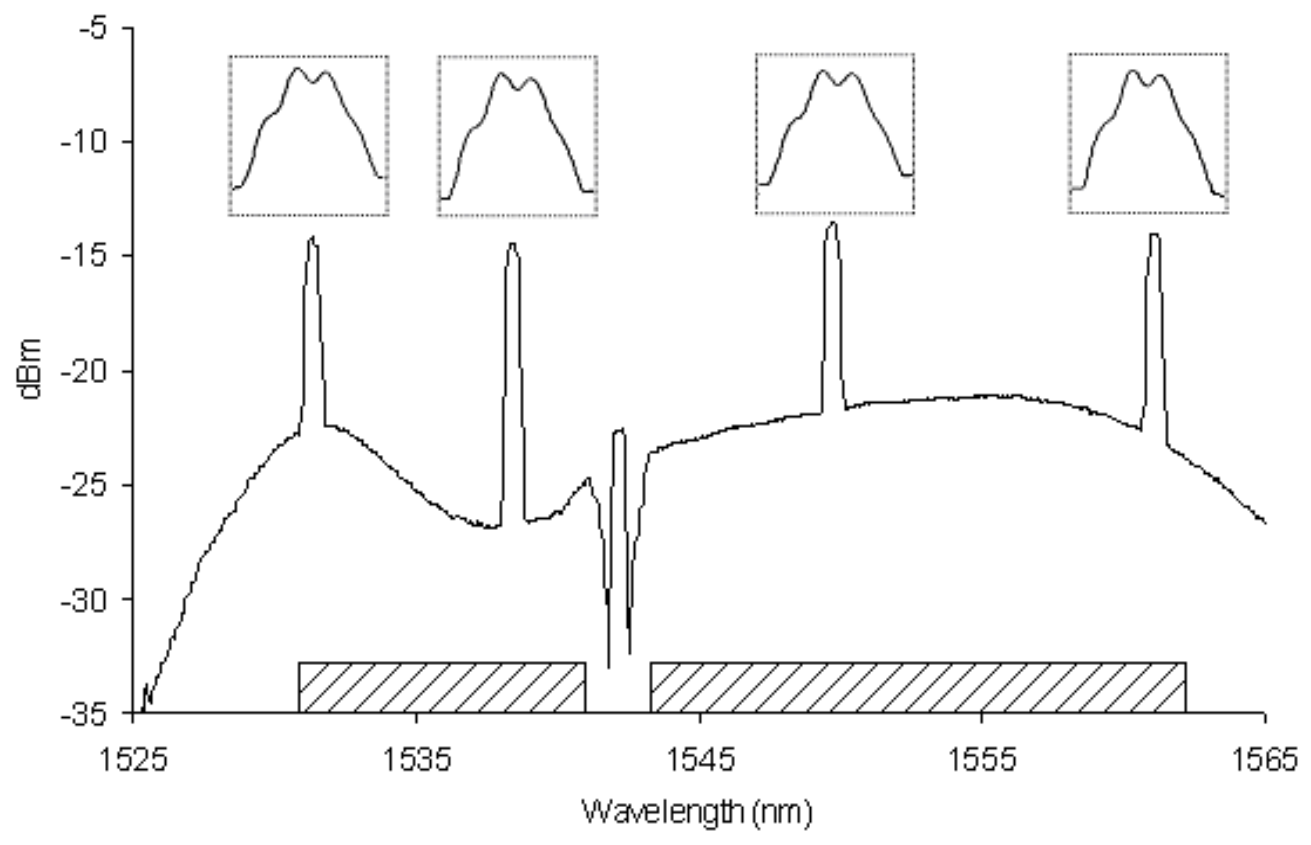


Figure 5

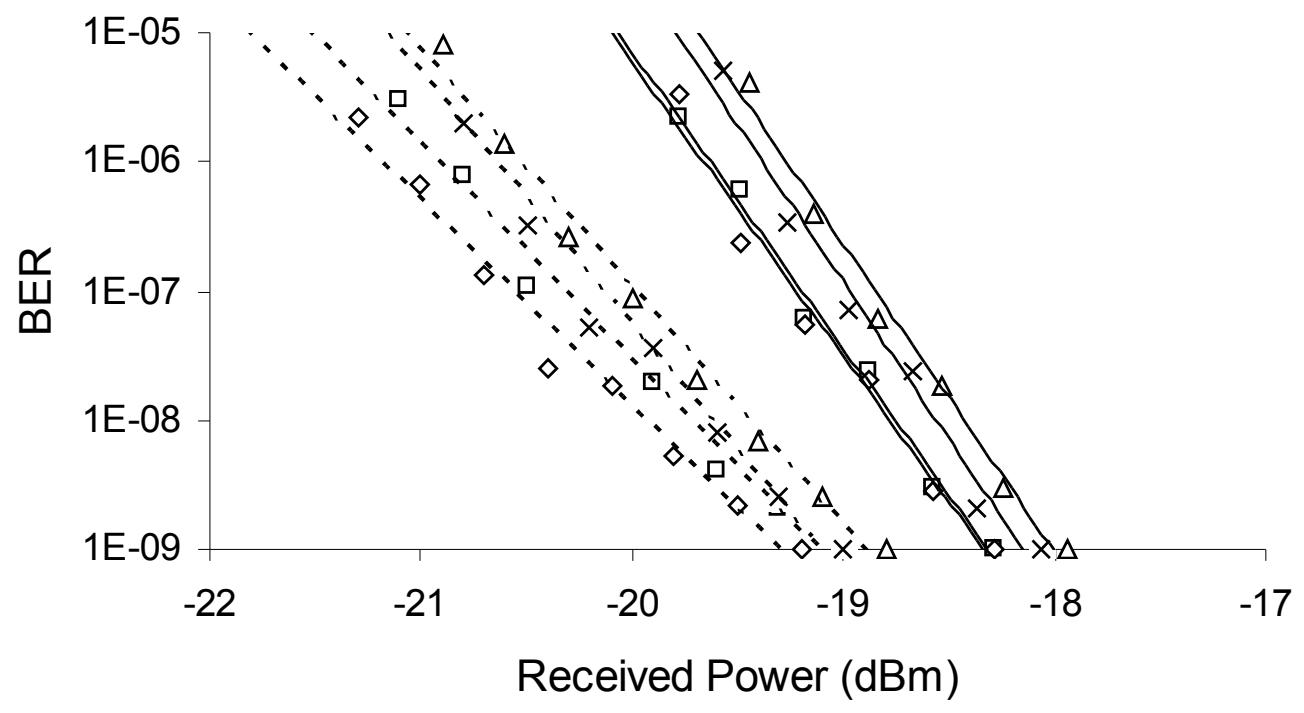

a

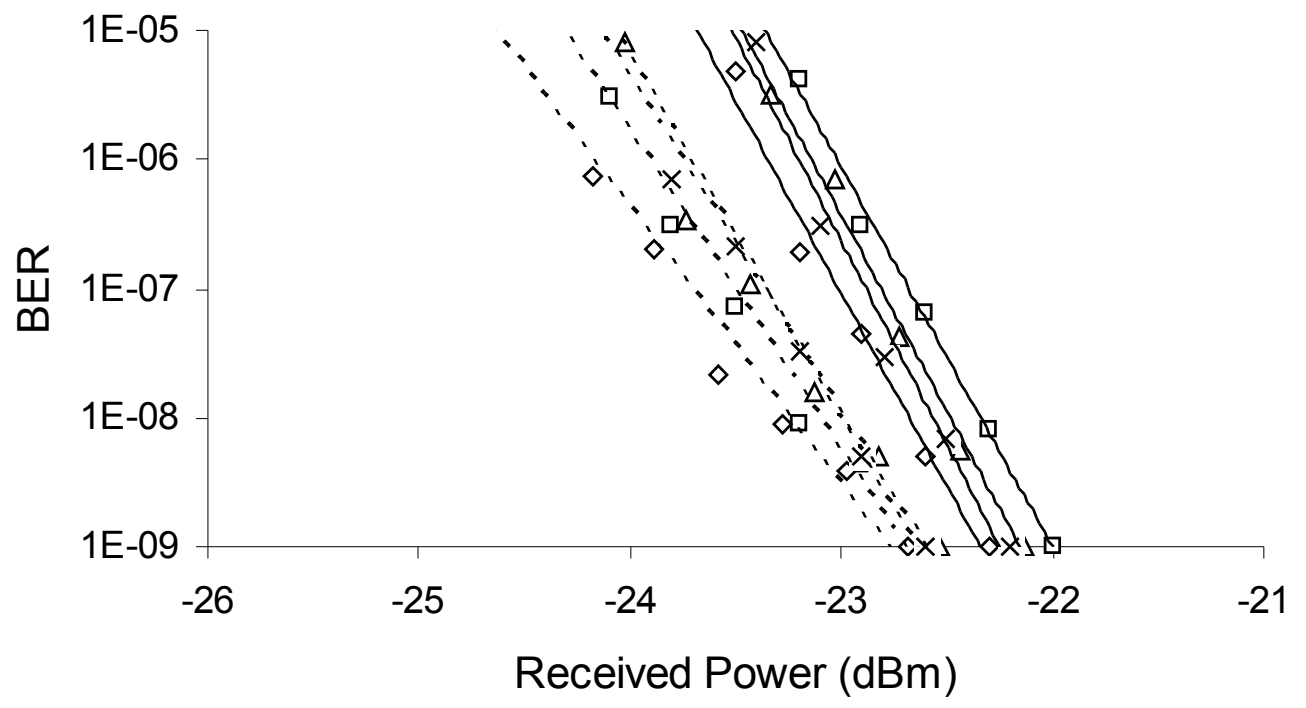

b 